\title{
Exploring a neural-network account of age-of-acquisition effects using repetition priming of faces
}

\author{
MICHAEL B. LEWIS, ANDREA J. CHADWICK, and HADYN D. ELLIS \\ Cardiff University, Cardiff, Wales
}

\begin{abstract}
The question of whether age-of-acquisition(AoA), frequency, and repetition priming effects occur at a common stage or at different stages of processing is addressed. Two single-stage accounts (i.e., cumulative frequency and a neural-network simulation) are considered in regard to their predictions concerning the interactions between AoA and frequency with aging and priming effects. A repetition-priming face-classification task was conducted on both older and younger participants to test these predictions. Consistent with the predictions of the neural-network simulation, AoA had an effect on reaction times that could not be explained by cumulative frequency alone. Also, as predicted by the simulation, the size of the priming effect was determined by the cumulative frequency of the item. It is discussed how this evidence is supportive of the notion that AoA, frequency, and priming all have effects at a common and single stage during face processing.
\end{abstract}

Words that are learned at a young age (e.g., school) are processed more quickly than those learned later in life (e.g., college). Evidence for this effect comes from experiments on picture-naming (Barry, Morrison, \& A. W. Ellis, 1997; Carroll \& White, 1973), word-reading (Gerhand \& Barry, 1998), and lexical-decision tasks (e.g., Gerhand \& Barry, 1999; Turner, Valentine, \& A. W. Ellis, 1998). The effects of age of acquisition (AoA) are found in addition to the effects of word frequency that are also found to influence the same set of tasks. The words that are read aloud fastest, are produced in an object-naming task, or are confirmed as being real words are those that are learned at a young age and have a high frequency in the adult environment.

The effects of AoA and frequency have also been found to generalize to face-related tasks (Moore \& Valentine, 1998), and these provide the focus of the research presented here. It is also important, however, to understand the issues surrounding AoA and frequency effects that have been primarily documented in lexical tasks. These are considered later, prior to our describing a face-categorization experiment.

There has been considerable interest in how one may account for the effects of frequency and AoA. The explanations can be roughly divided into two types: separatestage accounts (which suggest different loci of effects) and single-stage accounts (which suggest a common locus of effect). Historically, separate-stage accounts have been the dominant explanations. In the last few years, however, two

We thank A.W. Ellis and two anonymous reviewers for their useful comments on earlier versions of this paper. Correspondence should be addressed to M. B. Lewis, School of Psychology, Cardiff University, P.O. Box 901, Cardiff CF10 3YG, Wales (e-mail: lewismb@ cardiff. ac.uk). new explanationshave been offered for AoA and frequency effects, suggesting that, in fact, they share a common locus of effect. These explanations will be explored here, and, in particular, their predictions concerning effects of priming and aging will be developed. Once these predictions have been clarified, an experiment in which a face-classification task is used will be reported, the purpose of which was to establish which account is the more effective model of human performance.

\section{Separate-Stage Accounts}

Carroll and White (1973) reported the original finding relating the reaction times in a picture-naming task to AoA. They hypothesized that AoA, together with frequency (which was almost significant), could be a manifestation of the total number of times a word had been encountered. That is, AoA and word frequency have their effect at a common locus in the processing of lexical tasks. It would be expected, therefore, that the reaction times in these tasks would be predicted by some interaction between a word's frequency and how long it has been known (i.e., age of participant minus AoA). Carroll and White, however, failed to find such an interaction, and therefore, it has been concluded by others (e.g., Barry et al. 1997; Brown \& Watson, 1987) that AoA and frequency effects could not be explained by a single-stage account. Many subsequent studies of frequency and AoA effects have also failed to find an interaction between these factors (although there are some exceptions; e.g., Barry et al., 1997; Gerhand \& Barry, 1999), and so single-stage accounts of AoA effects have often been dismissed.

The rejection of the original single-stage accounts necessitated an explanation for the separate effects of frequency and AoA. Although frequency effects were rela- 
tively easy to account for, through gradual learning, a wide variety of accounts for an AoA effect on its own has been put forward. Suggestions have included (1) a push-down memory store (Carroll \& White, 1973), (2) lateralization of the development of language (H. D. Ellis \& Young, 1977), and (3) the break up of later-learned words to fit into a limited phonological store (i.e., phonologicalcompleteness hypothesis; Brown \& Watson, 1987). The latter explanation continues to enjoy popularity among researchers in the field (e.g., Barry, Hirsh, Johnston, \& Williams, 2001). One limitation of this account is that it predicts that AoA effects should occur only for tasks that require phonological retrieval. According to this account, tasks such as face categorization or recognition should not show AoA effects because they can be completed without access to phonology. There is an abundance of evidence, however, that AoA effects do occur in these tasks (see Lewis, 1999a; Moore \& Valentine, 1998, 1999). In order to reconcile this account with the face-related data, it would be necessary to propose a stage equivalent to phonological retrieval for face processing.

\section{The Revised Cumulative-Frequency Hypothesis}

The first of the recent single-stage accounts offered as an explanation of the AoA effect was a revision of the cumulative-frequency hypothesis that was originally rejected by Carroll and White (1973). The revised cumulativefrequency hypothesis (Lewis, 1999a) proposes that learning takes place by the accumulation of instances, where the total number of encounters (or instances) of a particular item will be determined by both its frequency and how long it has been known. The revision to the original hypothesis is that the reaction time to a stimulus is a power function of the number of times that it has occurred since the item was first encountered (cf. the power law of practice; Newell \& Rosenbloom, 1981). Lewis (1999a) demonstrated that the response time necessary to make a classification decision to a face (i.e., on which television program that person appeared) was determined by both the length of time that person had made regular appearances on the program and by his or her frequency of occurrence on the program when analyzed with the use of a power regression (i.e., analysis employing log transforms of independent and dependent variables). The validity of this explanation was challenged by Moore, Valentine, and Turner (1999), who expressed concern as to whether AoA effects and frequency effects show a dissociation. This challenge was responded to by Lewis (1999b), who demonstrated that there is no clear evidence for a dissociation between these factors.

Lewis, Gerhand, and H. D. Ellis (2001) reanalyzed a variety of English-language tasks that had originally been used to reject a cumulative-frequency account for AoA effects. In their survey paper, they showed how the nontransformed data violated assumptions of the analyses employed. The power regression analyses, however, were consistent with a cumulative-frequency account.
A further prediction of the cumulative-frequency hypothesis is that the size of the two effects of frequency and AoA should be equivalent. This is because each effect is produced through repetition so that one extra repetition attributable to increased frequency should be equivalent to one extra repetition attributable to one's having known the item for a longer time. This provides a further testable prediction of the cumulative-frequency hypothesis, a prediction that distinguishes this account from the second singlestage account considered here.

\section{The Neural-Network Account}

The neural-network simulations offered by A. W. Ellis and Lambon Ralph (2000) provide an alternative singlestage mechanism for how the relative effects of AoA and frequency might operate. By training a simple neural network in a manner that gradually built up the training set (in a similar manner to children's vocabulary development), they were able to show that both frequency effects and AoA effects are produced.

They used a three-layer back-propagation neural network to simulate the learning of words (although the items could represent any set of stimuli because the patterns that they used were random and abstract). Frequency and the time at which an item was introduced into the learning schedule were manipulated in order to investigate how the network's performance compared with that of humans on word tasks. Their network demonstrated a clear advantage for items that were introduced earlier into the network. This AoA effect was far beyond what would have been expected if performance was simply determined by cumulative frequency (even a power function of cumulative frequency). It was A. W. Ellis and Lambon Ralph's conclusion, therefore, that AoA effects are actually an automatic and emergent property of a neural network's performance when items are gradually introduced into the training set.

A. W. Ellis and Lambon Ralph (2000) offered an explanation for why the neural network shows such large AoA effects. The explanation was that, during "maturation" of the network, its plasticity is reduced. It is suggested that the hidden units lose plasticity during learning, leading to smaller changes taking place later in the learning schedule. The evidence for this comes from the fact that the hidden units show greater differentiation for early introduced items than for late-introduced items.

Although early or late entry into training cannot be reduced to simple differences in cumulative frequency (see Lewis, 1999a), frequency of presentation does have an effect on network performance: The more often a network is trained on a particular pattern, the stronger the representation becomes. Therefore, AoA effects should occur both in neural networks and in human adults whenever they are required to learn and represent associations in a cumulative and interleaved manner. Although A. W. Ellis \& Lambon Ralph's (2000) results do not accord with Lewis's (1999a) cumulative-frequency hypothesis (the neural network shows an effect of AoA that is far beyond that which 
can be accounted for by cumulative frequency), they cannot be used to rule out the possibility that AoA and frequency effects might be accommodated at a common stage of processing.

A further finding from the neural-network models of AoA effects is that the size of this effect is determined by the degree of consistency between inputs and outputs in such a way that the largest AoA effects are found where there is an arbitrary mapping (Anderson \& Cottrell, 2001; Smith, Cottrell, \& Anderson, 2001; Zevin \& Seidenberg, 2002). This prediction has found support in the analysis of consistent and inconsistent word naming: Monaghan and A. W. Ellis (2002) found that the effect of AoA was larger for inconsistent words (e.g., swarm) than for consistent words (e.g., swim). They also found, however, that the effect of frequency was larger for inconsistent words, again suggesting that the effects of frequency and AoA have common moderators. In the simulations and experiment in the present study, arbitrary mappings (random input-output mappings and face-TV program mappings, respectively) were used in order to show maximal AoA and frequency effects.

\section{Age or Order of Acquisition}

One main difference between the single-stage accounts and most separate-stage accounts is whether the important factor in the AoA variable is really the age of the subject, or whether it is merely the order of acquisition that is important. The phonological-completeness hypothesis suggests that there are developmental changes that take place as we learn language, and so, it really is the age of learning a word that produces the AoA effect. The cumulativefrequency hypothesis, however, suggests that age is irrelevant, and it is actually the length of time that something has been known (and hence the order in which it was learned) that produces an AoA effect.

Within the literature of lexical tasks, it is difficult to disentangle any potential effects of age of acquisition from effects of order of acquisition because the two factors are perfect correlates for words learned in the participant's first language. A study of second-language acquisition (e.g., Hirsh, Morrison, \& Carnicer, 2002) may be able to offer some solutions. It is far from clear, however, whether the second language shares a lexical and phonological store with the first; hence, conclusions from studies with bilinguals might not be conclusive.

Gilhooly (1984) conducted an experiment in which reaction times to newly invented words (e.g., skateboard) were studied in order to disentangle order of acquisition from AoA. His results suggest that the age of acquisition was more important than residence time (i.e., how long a word had been known). Problems with this study, however, include the fact that neologisms (e.g., skateboard) are more likely to be coined by and used by younger people, providing a possible confound.

With the use of famous faces as stimuli, rather than words, one is able to study participants of varying ages while keeping the time that stimuli are known the same for all participants. In this way, it might be possible to determine whether age of acquisition or time known is more important in determining reaction time.

\section{Priming Effects}

A further method that might be useful in distinguishing the various accounts of AoA effects is the use of repetitionpriming effects. Prior exposure to a face acts to reduce reaction times in tasks such as face naming (Valentine, Moore, \& Bredart, 1995), familiarity judgments (Bruce \& Valentine, 1985), and semantic classification(A. W. Ellis, Young, Flude, \& Hay, 1987; Lewis \& H. D. Ellis, 1999). The repetition-priming effect can be long lasting and is largest when exactly the same view of the primed individual is shown (Johnston \& Barry, 2001). Similar repetitionpriming effects also occur for word stimuli (e.g., Scarborough, Cortese, \& Scarborough, 1977), suggesting parallels between face processing and word processing.

Recently, Barry et al. (2001) explored how priming interacts with AoA and frequency effects in picture-naming and word-naming tasks. Four sets of stimuli were employed. Two sets were matched for imageability, name agreement, and frequency, but were either early acquired words or late-acquired words. The remaining two sets were matched on imageability, name agreement, and AoA, but were either high-frequency or low-frequency words. Using these four sets, they found a standard repetition-priming effect: Previously encountered items were produced more quickly. In addition, Barry et al. (2001) also found that the amount of priming interacted with AoA (so that later acquired items were primed to a greater degree), but it did not interact with the word frequency of the items.

The explanation offered by Barry et al. (2001) for their finding that AoA effects interact with priming involved their postulating separate stages for AoA and frequency effects. For late-acquired words, there is a greater amount of reaction time that can be saved by priming (i.e., initial, unprimed reaction times are much longer to begin with). The absence of an interaction between frequency and priming is taken as evidence that a frequency effect (which they dispute even exists for picture naming) must occur at a stage of processing other than lexical retrieval (where AoA is thought to have its locus).

Barry et al. (2001), however, also failed to find a significant word-frequency effect for either of their tasks. Frequency effects in lexical tasks have been found by various researchers-even when AoA effects have been controlled for (e.g., Brysbaert, Van Wijnendaele, \& De Deyne, 2000; A. W. Ellis \& Morrison, 1998; Gerhand \& Barry, 1998; Monaghan \& A. W. Ellis, 2002; Turner et al., 1998). Further, there is currently no theoretical framework that predicts that word frequency should not affect such tasks. Without a strong prediction that one should find no frequency effect, and given that others have found this effect, it is reasonable to conclude that the design employed by Barry et al. (2001) was not sufficiently sensitive to test for it.

Analysis of the items employed by Barry et al. (2001) supports the idea that the stimuli sets favored the finding 
of an AoA effect and not a frequency effect. Even though the ratio of the scores for frequency sets might be larger than that for the AoA sets, so too are the standard deviations. It is possible to use $z$ scores to compare the power of the contrasts between the four sets of stimuli employed. The $z$ score for the difference between the early and lateacquired sets of stimuli $(z=17.382)$ was considerably larger than that between the high- and low-frequency sets of stimuli $(z=6.360)$. Thus, the AoA manipulation was three times more powerful than the frequency manipulation. Since the effect of AoA might actually be larger than an equivalent frequency change (as predicted by the neural-network account), it is hardly surprising that no significant frequency effect was found.

This analysis of the stimuli sets leads to an alternative explanation for why no significant frequency $\times$ priming interaction was found by Barry et al. (2001). It is possible that the size of the priming effect observed was proportional to the reaction time of the unprimed stimulus. In effect, this was the explanation given by Barry et al. (2001) for the AoA $\times$ priming interaction. The effect of AoA is such that late-acquired words are produced more slowly than early acquired words, and so, one would expect to see more priming for late-acquired words-as, indeed, they did. The frequency manipulationemployed by Barry et al. (2001) failed to produce a significant effect on reaction time; thus low-frequency words were produced almost as fast as high-frequency words. If the size of the priming effect were determined by the reaction time of the unprimed stimuli, however, one would predict a small priming $X$ frequency interaction because the frequency effect itself is small. It is possible, therefore, that frequency, AoA, and priming all share the same locus. Indeed, such a conclusion is consistent with the data found by Barry et al. (2001), except that they failed to find any effects with their frequency manipulation.

\section{CONTRASTING THE PREDICTIONS OF THE ACCOUNTS}

From the discussion above, it is clear that there is a range of issues on which the separate-stage and the two singlestage accounts of frequency and AoA effects differ. The main difference between the cumulative-frequency hypothesis and the neural-network account is the relative sizes of AoA and frequency effects when analyzed in a power analysis. A second difference, moreover, is the relative size of AoA effects as a person gets older. The cumulativefrequency hypothesis suggests that, at any age, it is how long an item has been known that affects how efficiently it is recalled-and not the age at which learning has occurred. The loss-of-plasticity explanation of the neuralnetwork model suggests that as one gets older, the loss of plasticity will diminish, and so the effect of time known will decrease. This conclusion, however, is speculative. In order to determine exactly what A. W. Ellis and Lambon Ralph's (2000) neural network predicts about time-known effects and age, it is necessary to test the model. Such a test was performed and is reported below.

Barry et al. (2001) introduced the priming paradigm into the study of AoA effects. It is not obvious what the predictions of A. W. Ellis and Lambon Ralph's (2000) neural network are regarding priming. In order to establish its predictions, the simulation reported below was conducted. The prediction of the cumulative-frequency hypothesis regarding priming is quite simple: The more instances of an item, the smaller the priming advantage will be.

Barry et al. (2001) have offered some priming results with which to compare the predictions of the accounts. They concluded that the data are indicative of separate stages; however, from the discussion above, it can be seen that this conclusion comes from a null effect for frequency. Further evidence was sought regarding the relationship between time known (or AoA), frequency, and priming effects. This evidence was obtained by the use of a priming paradigm in the face-classification task mentioned above.

\section{Simulation}

In order to better understand the predictions made by A. W. Ellis and Lambon Ralph's (2000) neural-network model, a series of simple simulations was undertaken. These simulations were based on their neural networks, although a back-propagation neural network with no hidden units was employed. Initial pilot work in which both two-layer and three-layer networks were used revealed that either was capable of demonstrating the AoA and frequency effects described by A. W. Ellis and Lambon Ralph. This point, in itself, suggests problems for the "loss of plasticity" explanation of AoA effects because it has been supposed that it is the plasticity of the hidden units that is lost. It is necessary, therefore, to be able to explain the AoA effects observed in neural networks without the use of hidden layers. The two-layer network used here is simpler than their three-layer version, and so, by application of Occam's razor, it may be more appropriate.

Aging. The network was trained in two ways: one that represented younger participants, with test items' entering the training schedule from the second block of trials, and another that represented older participants in whom the network has already learned many background items that were different from the test items. These background items varied in their frequency, and the network was continually trained on them-even after the test items had been introduced. The same background items were also presented to the young network along with the test items.

The test stimuli were 160 randomly generated arrays of 200 zeros or ones. The first 100 units of each pattern represented the input, and the second 100 units represented the target output. The zeros and ones were generated by using a binomial distribution $(n=1, p=.5)$. The stimuli were divided factorially into four levels of frequency (the relative frequencies were 1,2,4, and 8) and four levels of AoA (items were introduced in Blocks 2, 3, 4, and 5). 
There were 10 stimuli in each of the frequency $\times$ AoA cells. The background stimuli were generated in a similar random manner.

An "older" network was generated by pretraining the network on the background items for 31 blocks prior to introducing any of the test items (these blocks are numbered from -29 to 1 ). The background items included 160 items, and they varied in their frequency within each block so that they were presented one, two, four, or eight times. The "younger" network was trained on the background items for just 1 block. In each block, the network was trained on all items in the training schedule 10 times. During Block 1 (and the blocks previous to that for the older network), only the background items were included in the learning environment. During Block 2, the 40 items with AoA values of 2 were repeated during each epoch according to their particular relative frequency in addition to the background items. During each successive block, a new set of items was introduced into the learning environment until all five sets had been introduced by the Block 5 occurred.

The performance of the models after Block 5 was measured and recorded. The measure of performance error for each item was recorded as the sum of the squares of the differences between each output unit and its target output (pattern sum of squares, or pss). This is the same performance measure used by A. W. Ellis and Lambon Ralph (2000).

The performance of the two networks is illustrated in Figure 1 . The overall performance was not significantly different between the younger and older networks, suggesting that any overall age differences in humans must be modeled by parameter changes rather than by environmental changes to the network. An analysis was conducted on the error to the trained items after Block 5. The dependent variable was the log transform of the error term (pss; although similar results were found for the analyses with the nontransformed pss). The independent variables were the log of how many blocks in which the item had been presented (i.e., log of time known) and the log of the frequency of the items in the blocks that they were in (i.e., $\log$ of frequency). This method was used because it allowed the data to be accounted for by cumulative frequency, and any further AoA effect would appear by way of a larger time-known coefficient than frequency coefficient.

The frequency coefficient was significant for both the younger network and the older network $(p s<.0001)$, and the coefficients were -.151 and -.145 , respectively,

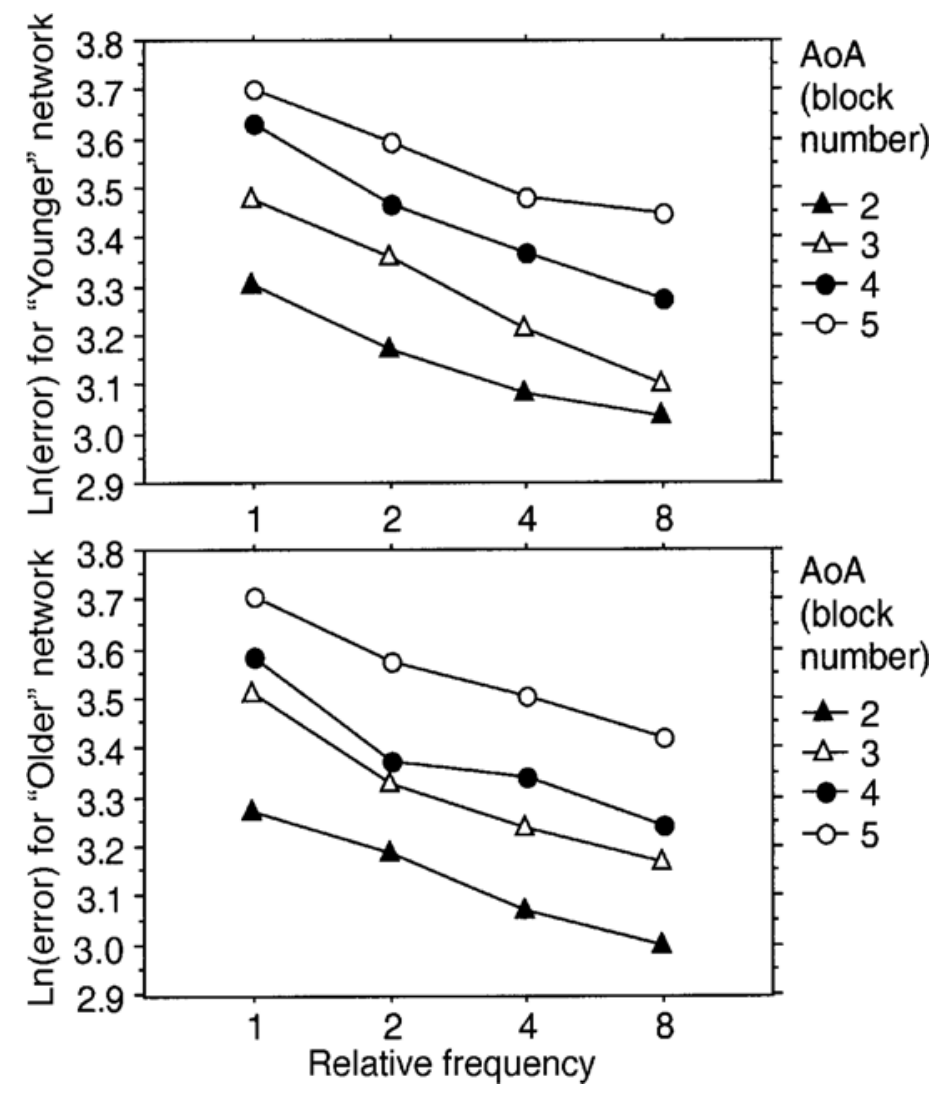

Figure1. Performance after Block 5 of younger and older neural-network simulations on the test items, according to item frequency and item age of acquisition (AoA). 

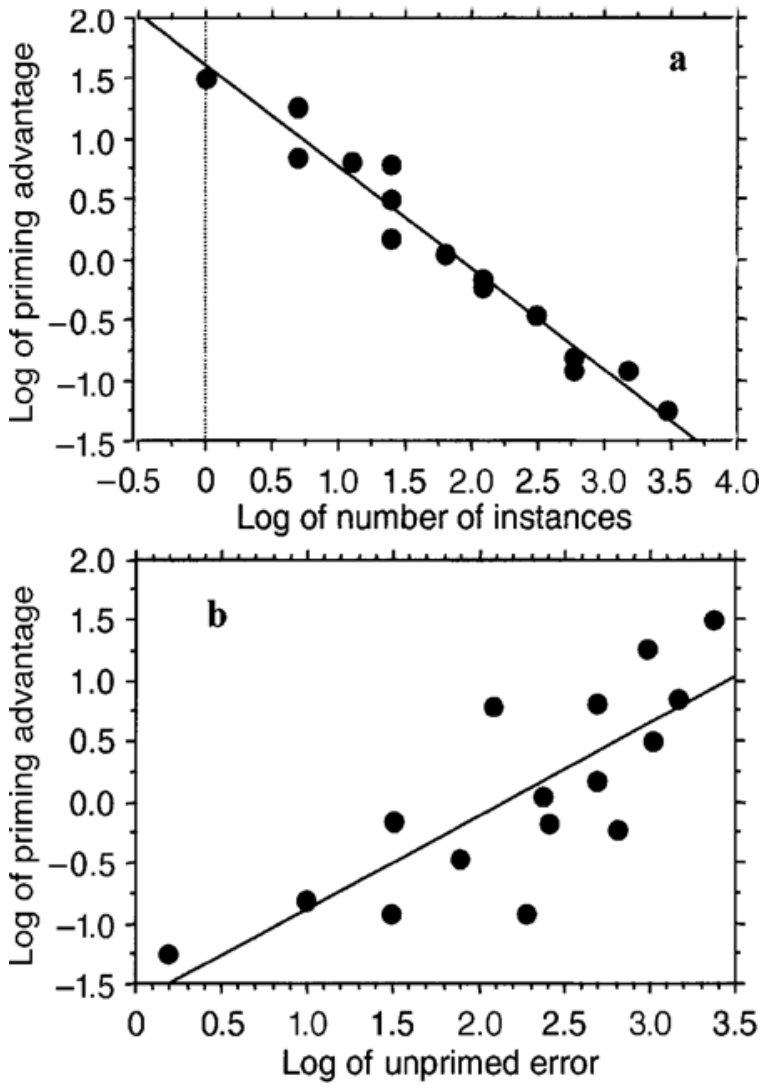

Figure 2. The priming advantage found in the younger neural network as predicted by (a) $\log$ of number of previously trained instances of each item, and (b) the log of the error when the item was not primed.

which were not significantly different from each other. Frequency, therefore, appears to affect the learning of new items in the younger and older networks in the same way. Each of the time-known coefficients was also significant $(p \mathrm{~s}<.0001)$ and was also significantly more negative than its respective frequency coefficients $(p s<.0001)$. The time-known coefficient for the younger network was -.286 , and the coefficient for the older network was -.282 . The model predicts, therefore, that there is a timeknown (or AoA) effect beyond that attributable to cumulative frequency, and, further, the size of this effect is independent of age. Independence of age is consistent with the findings of Morrison, Hirsh, Chappell, and A. W. Ellis (2002), who found that the size of an AoA effect in objectnaming or word-reading tasks was not influenced by the age of the subject.

Priming. Priming was modeled on the younger network only by comparing the performance for all 160 test items at a particular point in the learning sequence with performance when the network had been trained on one extra presentation of each item. A comparison was made of the network's performance immediately prior to and following this extra learning, or priming, phase. The difference in the performance was calculated and is referred to as the prim- ing advantage. The priming advantage was subsequently analyzed to determine what factors determine its size.

The priming advantage shown in Figure 2 displays a good fit to a power function of the number of learning instances of that item. The priming advantage, however, does not show a good a fit to the simple unprimed error. An analysis was conducted to determine what factors would significantly predict the priming advantage. The $\log$ transform of the priming advantage (with an amount added to prevent loss of negative data- a similar addition was found to be required for the human data; see below) was regressed against log of number of instances, log of error to unprimed stimuli, and log of frequency (this final variable would reveal whether there was any frequency or AoA effect beyond the cumulative-frequency, or instances, variable). The only predictor that was significant was the $\log$ of the number of previous instances of that item $\left[R^{2}=\right.$ $.971, b=-.696 ; t(12)=3.886, p<.0001]$. The log of frequency was not significant $[b=-.195 ; t(12)=1.477$, $p>.05]$, nor was the predictive power of the log of the unprimed error, once the other two predictors had been considered $[b=.019 ; t(12)=0.147, p>.05]$.

The size of the priming advantage, as predicted by the A. W. Ellis and Lambon Ralph (2000) model, is accounted for by one fact alone: The total number of times the item has been seen previously will act to reduce the priming advantage. The effects of AoA and frequency, therefore, will combine to produce a cumulative-frequency prediction regarding the size of the expected priming effect. This prediction was tested in the experiment described below. One of the other predictions of the model, that was tested was the relative magnitude of AoA and frequency effects. The main contrast between this neural-network model and the cumulative-frequency hypothesis is that the latter predicts equivalent magnitudes of the two effects when they are interpreted as number of repetitions.

\section{EXPERIMENT}

A face-classification experiment was conducted to test a range of questions regarding the roles played by AoA, frequency, age, and priming. In the experiment, we employed a task in which participants had to report whether the presented faces were of characters from one of two soap operas. This experiment, therefore, was similar to that reported by Lewis (1999a), but there were a number of important differences. The first was that only current characters were employed as stimuli. This adjustment meant that it was not necessary to consider increases in reaction times brought on by the long-term forgetting of people. In addition, the problem of actors' being known for their roles in subsequent programs would not have to be considered here. The second difference was that participants of varying ages were tested. This manipulation was included to distinguish between age of acquisition and order of acquisition. The third difference was that there was a priming component to the experiment. For each participant, half of the faces were repeated to contrast primed and unprimed reaction times. 


\section{Method}

Participants. Two groups of participants were selected on the basis of age and were drawn from the Cardiff University participant panels. The 32 younger participants had a mean age of 20.4 years (range 18-25 years), whereas the 31 older participants had a mean age of 60.4 years (range $41-80$ years). All participants fulfilled the criterion that they were regular viewers (at least once a week) of both TV shows from which the stimuli came All the participants had normal or corrected-to-normal vision.

Stimuli. Eighty-six face-only views of characters from the BBC's "Eastenders" (34 faces) and Granada's "Coronation Street" (52 faces), the two most-watched television soap operas shown in the U.K., were used as stimuli. All characters were current members of the casts of the TV shows and had appeared within the last 2 months. For each face, the total length of time (in months) that the character had appeared on the show was recorded. Further, a frequency of occurrence measure was also obtained. This information was obtained by counting the number of times each character's name occurred in synopses of plot written over a 3-year period, published on fan web sites. These synopses were written by fans soon after the broadcast of each episode and provide a summary of the activities of characters during the program. Four episodes of each program were viewed and compared with the fan-generated synopsis. This showed a strong correlation between the length of time the characters were on the screen and the number of times they were mentioned in the synopsis. The frequency measure was generated by adjusting for the length of time the person had been on the show, and the measure was normalized between the two programs. This measure may be noisy, but it is useful because it is objective. The 86 faces were divided into two sets: $a$ and $b$. A pilot study that measured the average reaction time for categorization of each face was conducted counterbalance the performance for faces between the two sets using an ABBA method.

Procedure. The participants were presented with a series of faces and were required to respond quickly, using one of two keys to indicate in which TV show each character appeared. The faces were displayed until a response was made. If the participant did not recognize the face, he or she was encouraged to press a third key rather than guess.

The faces were presented in two phases (although this was not apparent to the participants, since the phases ran into each other). Half of the participants were presented with Set $a$ in Phase 1, followed by Sets $a$ and $b$ in Phase 2. The remaining participants were presented with Set $b$ in Phase 1, followed by Sets $a$ and $b$ in Phase 2. The faces in each phase were presented in a different randomized order for each participant.

Design. The dependent variable was the reaction time required for the participants to categorize each character correctly. The independent variables were the length of time each character had appeared on the show, the frequency of each character's appearance on the show during that time, and the participant's age (older vs. younger adult).

\section{Results}

In order to explore two separate issues of age or order of acquisition and the effects of priming, the data were ana- lyzed in two ways. First, the reaction times were considered for responses made the first time that the participants saw each face (i.e., unprimed data). The two different conditions that the participants were in ( $a$ then $a b$ or $b$ then $a b$ ) was irrelevant for this analysis since this just served to counterbalance order of presentation. The second analysis was done on the degree to which priming reduced the average reaction time in classifying an item (i.e., the priming effect). This analysis compared the unprimed reaction times from one set of participants with the primed reaction times of the other set of participants.

Unprimed data. A comparison between the two groups of participants revealed that the younger participants were significantly faster at the task than the older participants [younger mean $\mathrm{RT}=1,011 \mathrm{msec}$, older mean $\mathrm{RT}=1,684$ msec $;(85)=18.221, p<.0001]$. A multiple-regression analysis was conducted on the unprimed data by using both the by-item and the by-participant methods (Lorch \& Myers, 1990). In each analysis, the main independent variables were (1) the log transform of the period of time that each character had appeared on the TV show [ln(time known)] and (2) the log transform of the mean frequency for the character over the previous 3 years $[\ln (f r e q)]$. Separate analyses were conducted on the data for the older and younger participants. The dependent variable was the $\log$ transform of the time it took to categorize correctly the character of one of the two TV shows. The transformation ensured that the equivalance of the size of the effects could be meaningfully compared (see Lewis, 1999a). The errors and any stimuli not known by the participant were removed from the analysis (these made up less than $1 \%$ of responses). The by-items analysis was globally significant for the younger group $\left[R^{2}=.434 ; F(2,83)=31.821, p<\right.$ $.0001]$ and the older group $\left[R^{2}=.415 ; F(2,83)=29.390\right.$, $p<.0001]$. The regression coefficients are shown in Table 1. Each of the independent factors was significant for the younger group [ln(time known): $t(83)=6.897$, $p<.0001$; $\ln ($ freq) $: t(83)=5.084, p<.0001]$. These factors were also significant for the older group [ln(time known): $t(83)=7.168, p<.0001 ; \ln ($ freq $): t(83)=$ $3.855, p<.001]$. For the by-participants analysis, both factors were significant for the younger group [ $\ln ($ time known): $t(32)=12.996, p<.0001 ; \ln ($ freq $): t(32)=$ $8.370, p<.0001]$. For the older group, each factor was also significant $[\ln ($ time known): $t(31)=16.145, p<$ $.0001 ; \ln$ (freq): $t(32)=5.597, p<.0001]$.

Although the analysis described so far illustrates that both time known and frequency affect the reaction time, it has not addressed the relative size of the coefficients. In order

Table 1

Regression Coefficients for the Two Main Variables for Each Set of Participants

\begin{tabular}{|c|c|c|c|}
\hline \multirow[b]{2}{*}{ Variables } & \multicolumn{2}{|c|}{ Regression Coefficients } & \multirow{2}{*}{$\begin{array}{l}\text { Significant } \\
\text { Difference? }\end{array}$} \\
\hline & Younger Subjects & Older Subjects & \\
\hline Ln(time known) & $-.044 *$ & $-.076^{*}$ & Yes* \\
\hline $\operatorname{Ln}($ frequency) & $-.055^{*}$ & $-.068 *$ & No \\
\hline Significant difference? & No & Yes** & \\
\hline
\end{tabular}


to do this, direct comparisons must be made between the coefficient estimates generated from the by-items and byparticipants analyses. For the younger participants, the coefficient for $\ln ($ freq) was slightly more negative than that for $\ln$ (time known), but this difference was not significant. For the older participants, the coefficient for $\ln ($ time known) was more negative than that for $\ln ($ freq), and this difference was significant. Comparisons could also be made between the regression coefficients for the older and younger participants. When the comparison was made for coefficients of $\ln ($ freq), no significant difference was found $[t(63)=0.116, p>.05]$. The coefficients of $\ln ($ time known $)$ were more negative for the older participants than for the younger participants - a difference that was highly significant $[t(63)=4.726, p<.001]$.

Priming effects. In order to analyze the effect of priming, mean reaction times for correct categorization of each face, both when primed and when not primed in Phase 2 of the experiment, were compared. Paired $t$ tests revealed a significant priming advantage both for the younger [mean difference $=158 \mathrm{msec} ; t(85)=9.324, p<.0001$ ] and for the older [mean difference $=277 \mathrm{msec} ; t(85)=$ $9.256, p<.0001]$ participants. The data were subsequently analyzed in order to test the relationship among primed and unprimed reaction times, simple frequency, and cumulative frequency, as predicted by the neural-network simulation above.

Multiple regressions were conducted for the older and younger participants on the log of the mean difference in primed and unprimed categorization times $(200 \mathrm{msec}$ were added to all the differences because this prevented many of the negative priming effects' being removed from the dataset when logged-this was similar to how the neural-network priming data were handled). The predictors considered were (1) the log of the total number of instances of a face calculated by the frequency multiplied by time known, (2) the log of the frequency itself (which would reveal any frequency or AoA effects beyond a cumulative-frequency explanation), and (3) and the log of the unprimed reaction time (taken as a mean for the items when presented in Phase 1 of the experiment).

For the younger participants, there was a significant effect of $\log$ of instances $[b=-.083 ; t(81)=2.942, p>$ $.01]$. The predictor of log of frequency was in the opposite direction to that predicted but was not significant $[b=$ $.007 ; t(81)=0.144, p>.05]$. The effect of the log of the unprimed reaction time was not significant when the other factors were considered $[b=.301 ; t(81)=0.755, p>$ $.05]$. For the older participants, none of the predictors was significant, but the effects of log of instances and log of frequency were in the same direction as those for the younger participants. The predictor closest to significance was $\log$ of number of instances $[b=-.039 ; t(81)=1.468$, $p=.146]$.

Summary of results. The data show that, for both the older and the younger participants, the length of time for which a person is known and their frequency of occurrence will predict reaction time in a face-classification task. For the younger participants, the size of these two ef- fects is similar, but for the older participants, time known makes a larger contribution than does frequency. The size of a priming effect is predicted by the total number of times that a person has been seen before.

\section{Discussion}

The experiment described here yields a range of potentially important and interesting results. These results require careful consideration in the light of the literature on AoA, frequency, and priming effects as well as the neuralnetwork simulation reported above.

Time known and frequency coefficients. The first interesting finding is that, for the younger participants, both AoA and frequency effects predicted the speed of face categorization, so early acquired high-frequency faces were categorized fastest. The coefficients for these two effects are almost identical-a result that is consistent with the findings reported by Lewis (1999a) and supportive of the cumulative-frequency effect. The older participants also showed effects of time known and frequency. For these participants, however, the size of the coefficient for time known was significantly larger than that for frequency. This finding is not consistent with the cumulative-frequency hypothesis but, instead, is consistent with the A. W. Ellis and Lambon Ralph (2000) account (and with the neuralnetwork simulation above), which predicts larger coefficients for time-known effects than for frequency effects at all ages.

Although it might be possible to dismiss the lack of a difference between the coefficients for the younger participants as a null result, the fact that there was a significant difference between the older and younger participants means that this result needs to be explained. It appears that there was something that amplified the frequency effects relative to the time-known effects for the younger participants but that was absent for the older participants. It is possible that the younger participants had changed their viewing habits over the period considered in the timeknown variable. If these younger participants had increased their frequency of viewing, this would have resulted in a smaller time-known effect, since the benefit of being seen earlier is offset by a lower frequency over the character's time on the series. This explanation would account for the pattern of effects observed here (and reported by Lewis, 1999a), although still being consistent with the A. W. Ellis and Lambon Ralph (2000) account. It is possible, however, that the effect observed was not a methodological artifact but, in fact, indicates that older people do show larger AoA effects. Such a finding is inconsistent with all of the accounts of AoA effects that have been put forward, and so this effect, if replicable, could be of importance.

However one accounts for the effects in the younger participants, the older participants clearly demonstrated that the effects of time known and frequency in a faceclassification task do not simply reduce to cumulative frequency. This conclusion is in contrast to that drawn by Lewis (1999a). Evidence from the younger participants in the present experiment shows why the original cumulativefrequency hypothesis could be invoked (these participants 
showed equivalence of time-known and frequency effects as predicted by this hypothesis); but the older participants showed that the equivalence of coefficients (for time known and frequency) was not a robust finding. The preferred explanation of the effects observed here (and in the experiment reported by Lewis, 1999a) is that there is a cumulative-frequency effect and an order-of-acquisition effect that act beyond the cumulative frequency-that is, the results are consistent with the A. W. Ellis and Lambon Ralph (2000) account and not with the cumulative-frequency hypothesis.

Priming effects. The investigationinto priming effects reveals clear results. First, there was a significant effect of priming - faces that were seen before were responded to faster than were those that had not been seen before. Further, the sole significant determinant of the size of the priming effect was the number of instances that a face had been previously encountered. No other factor, be it unprimed reaction time, AoA, time known, frequency, or any other variation of these, made a further significant contribution to explaining the size of the priming effect.

The influence of the number of instances on the size of the priming effect was a prediction of the neural-network hypothesis presented here. The empirical data, therefore, coincide with the prediction generated from the implementation of the A. W. Ellis and Lambon Ralph (2000) model. This result, however, is also consistent with the prediction of the cumulative-frequency hypothesis-but this hypothesis was not found to be consistent with the evidence described above and so will not be considered further here.

It was described above how the less negative time-known component found to predict classification reaction time could have been an artifact caused by changes in the younger participants' viewing habits. One might expect a similar reduction in the negativity of the time-known coefficient in the analysis of the priming task for a similar reason. A consequence of this is that a residual AoA effect beyond number of instances might have been lost from the data. Therefore, of course, we cannot reject the possibility of there being an additional effect of AoA on priming, but the present experiment did not find it.

Barry et al. (2001) found that the AoA of the words that they used influenced the size of a priming advantage but they found no significant effect for their frequency measure. On the contrary, in the experiment presented here it was the total number of instances of a face that predicted the priming advantage. These different results can be reconciled if it is indeed the number of instances that predicts priming - as is predicted by the A. W. Ellis and Lambon Ralph (2000) neural network. From the Barry et al. (2001) data, it can be seen that the early and late-acquired words are more different from each other than are the high- and low-frequency words (in terms of the $z$ scores of the sets as described above). Such a difference would explain why Barry et al. (2001) found that AoA and not frequency affected the priming advantage - this difference, however, would be no larger than the difference between the two effects observed on simple reaction times (which they also reported to be affected by AoA and not by frequency). According to this interpretation, it can be predicted that wherever one finds a frequency or AoA effect, it should interact with priming.

Although our data coincide with the predictions of the model, it is difficult to draw theoretical conclusions, not least because of the high degree of multicolinearity. The model predicts that the number of instances will determine the size of the priming advantage, but this is highly correlated with the performance on unprimed items (unprimed items are predicted by instances and by an additional time-known component). Determining whether it is really the number of instances or, instead, the unprimed performance that actually predicts the priming advantage will be extremely difficult because the two are intricately linked. It is sufficient here to observe that human performance on the priming task can be modeled by the neural network. Our understanding of the full theoretical implications of this model remains to be achieved. A. W. Ellis and Lambon Ralph (2000) discussed the model in terms of loss of plasticity. How such a concept can be used to explain how AoA and frequency effects affect priming remains to be seen. One theoretical conclusion that can be drawn from the neural-network and empirical research presented here is that a two-layered neural network can model all the effects of AoA, frequency, and priming. There is no need, therefore, to consider these three effects to have separate loci-at least for the processes involved in face classification.

\section{SUMMARY AND CONCLUSIONS}

The neural-network simulation and face-classification experiment, together, have offered a clearer insight into how the factors of age, AoA (or time known), item frequency, and repetition priming interact. The neural network enabled a number of predictions to be formulated. When reaction time was analyzed with the use of power regression methods, it was predicted that (1) the size of the time-known coefficient would be more negative than the frequency coefficient, (2) the size of these coefficients would be independent of age of the participant, and (3) the size of a priming advantage could be predicted by the total number of instances of that particular item. The first of these predictions is in contrast to the predictions of the cumulative-frequency hypothesis (Lewis, 1999a; Lewis et al., 2001). The last of these predictions is in contrast to those of separate-stage accounts (Barry et al., 2001).

A face-classification task was analyzed in order to test these predictions. The first and third of these predictions were found to be supported by the participants' performance on the task. The second prediction was contradicted by the data, but we cannot discount the possibility that this was due to a methodological problem. These data, therefore, provide evidence against the cumulative-frequency hypothesis and support the account offered by A. W. Ellis and Lambon Ralph (2000) that is based on a neural-network model. Further, the work reported here illustrates that AoA effects, frequency effects, and priming effects can si- 
multaneously occur at a common and single stage of processing, in contrast to the conclusions drawn by Barry et al. (2001) that were based on lexical tasks.

The question may be asked, at what stage of processing do AoA, frequency, and priming have their influence? It has been shown here (and elsewhere; e.g., A. W. Ellis \& Lamdon Ralph, 2000) that effects that have been used to postulate separate stages can, with the use of neuralnetwork models, be incorporated into a single-stage model. The desire to attribute effects to distinct and describable stages is a legacy of functional models of cognition. The neural-network simulation presented here demonstrates that we can expect to find AoA (or order of acquisition) effects, frequency effects, and repetition priming effects wherever an arbitrary mapping is learned though an incremental process. Whatever stages of processing one wishes to propose, if they involve an arbitrary mapping (as in most cases they do), we can predict that order, frequency, and priming effects will occur. Although ultimately testing these predictions is an empirical issue, the arbitrary-mapping hypothesis makes clear predictions about where the effects of order of learning, frequency, and priming should be found as well as the relative size of these effects.

\section{REFERENCES}

Anderson, K. L., \& Cottrell, G. W. (2001). Age of acquisition and connectionist networks. In Proceedings of the Twenty-Third AnnualMeeting of the Cognitive Science Society (pp. 27-32). Mahwah, NJ: Erlbaum.

Barry, C., Hirsh, K. W., Johnston, R. A., \& Williams, C. L. (2001). Age of acquisition, word frequency, and the locus of repetition priming of picture naming. Journal of Memory \& Language, 44, 350-375.

Barry, C., Morrison, C. M., \& Ellis, A.W. (1997). Naming the Snodgrass and Vanderwart pictures: Effects of age of acquisition, frequency, and name agreement. Quarterly Journal of Experimental Psychology, 50A, 560-585.

Brown, G. D. A., \& Watson, F. L. (1987). First in, first out: Word learning age and spoken word frequency as predictors of word familiarity and word naming latency. Memory \& Cognition, 15, 208-216.

Bruce, V., \& VAlentine, T. (1985). Identity priming in the recognition of familiar faces. British Journal of Psychology, 76, 363-383.

Brysbaert, M., Van Wijnendaele, I., \& De Deyne, S. (2000). Ageof-acquisition effects in semantic processing tasks. Acta Psychologica, 104, 215-226.

Carroll, J. B., \& White, M. N. (1973). Word frequency and age of acquisition as determiners of picture naming latencies. Quarterly Journal of Experimental Psychology, 12, 85-95.

Ellis, A. W., \& Lambon Ralph, M. A. (2000). Age of acquisition effects in adult lexical processing reflect loss of plasticity in maturing systems: Insights from connectionist networks. Journal of Experimental Psychology: Learning, Memory, \& Cognition, 26, 1103-1123.

Ellis, A. W., \& Morrison, C. M. (1998). Real age-of-acquisition effects in lexical retrieval. Journal of Experimental Psychology: Learning, Memory, \& Cognition, 24, 515-523.

Ellis, A. W., Young. A. W., Flude, B. M., \& Hay, D. C. (1987). Repetition priming of face recognition. Quarterly Journal of Experimental Psychology, 39A, 193-210.

Ellis, H. D., \& Young, A. W. (1977). Age-of-acquisition and recognition of nouns presented in the left and right visual fields: A failed hypothesis. Neuropsychologia, 15, 825-828.

GERHAND, S., \& BARRY, C. (1998). Word frequency effects in oral reading are not merely age-of-acquisition effects in disguise. Journal of
Experimental Psychology: Learning, Memory, \& Cognition, 24, 267283.

GERHAND, S., \& BARRY, C. (1999). Age of acquisition, word frequency, and the role of phonologyin the lexical decision task. Memory \& Cognition, 27, 592-602.

GiLhooly, K. J. (1984). Word age-of-acquisition and residence time in lexical memory as factors in word naming. Current Psychological Research \& Reviews, 3, 24-31.

Hirsh, K. W., Morrison, C. M., \& Carnicer, E. (2002). Age of acquisition and speech production in L2. Manuscript submitted for publication.

JoHnSton, R. A., \& BARRY, C. (2001). Best face forward: Similarity effects in repetition priming of face recognition. Quarterly Journal of Experimental Psychology, 54A, 383-396.

LEWIS, M. B. (1999a). Age of acquisition in face categorisation: Is there an instance-based account? Cognition, 71, B23-B39.

LEWIS, M. B. (1999b). Are age-of-acquisition cumulative-frequency effects in disguise? A reply to Moore, Valentine and Turner (1999). Cognition, 72, 311-316.

LewIS, M. B., \& Ellis, H. D. (1999). Repeated repetition priming in face recognition. Quarterly Journal of Experimental Psychology, 52A, 927-955.

Lewis, M. B., Gerhand, S., \& ElLis, H. D. (2001). Re-evaluating age of acquisition effects: Are they simply cumulative-frequency effects? Cognition, 75, 1-17.

LoRCH, R. F., \& Myers, J. L. (1990). Regression analysis of repeated measures data in cognitive psychology. Journal of Experimental Psychology: Learning, Memory, \& Cognition, 16, 149-157.

Monaghan, J., \& Ellis, A. W. (2002). What, exactly, interacts with spelling-sound consistency in word naming? Journal of Experimental Psychology: Learning, Memory, \& Cognition, 28, 183-206.

Moore, V., \& VAlentine, T. (1998). The effect of age of acquisition on speed and accuracy of naming famous faces. Quarterly Journal of Experimental Psychology, 51A, 485-513.

Moore, V., \& VAlentine, T. (1999). The effects of age of acquisition in processing famous faces and names: Exploring the locus and proposing a mechanism. In Proceedings of the Twenty-First Annual Meeting of the Cognitive Science Society (pp. 416-421). Mahwah, NJ: Erlbaum.

Moore, V., Valentine, T., \& Turner, J. (1999). Age-of-acquisition and cumulative frequency have independent effects. Cognition, 72, 305310 .

Morrison, C. M., Hirsh, K. W., Chappell, T., \& Ellis, A. W. (2002). Age and age of acquisition: An evaluation of the cumulative frequency hypothesis. European Journal of Cognitive Psychology, 14, 435-459.

Newell, A., \& Rosenbloom, P. S. (1981). Mechanisms of skill acquisition and the law of practice. In J. R. Anderson (Ed.), Cognitive skills and their acquisition (pp. 1-55). Hillsdale, NJ: Erlbaum.

Scarborough,D. L., Cortese, C., \& Scarborough,H. S. (1977). Frequency and repetition effects in lexical memory. Journal of Experimental Psychology: Human Perception \& Performance, 3, 1-17.

Smith, M. A., Cottrell, G. W., \& Anderson, K. L. (2001). The early word catches the weights. In T. K. Leen, T. G. Dietterich, \& V. Tresp (Eds.), Advances in neural information systems 13 (pp. 52-58). Cambridge, MA: MIT Press.

Turner, J. E. Valentine, T. \& Ellis, A. W. (1998). Contrasting effects of age of acquisition and word frequency on auditory and visual lexical decision. Memory \& Cognition, 26, 1282-1291.

Valentine, T., Moore, V., \& Bredart, S. (1995). Priming production of people's names. Quarterly Journal of Experimental Psychology, 48A, 513-535.

Zevin, J. D., \& Seidenberg, M. S. (2002). Are there independent ageof-acquisition effects in word reading? Journal of Memory \& Langauge, 47, 1-29.

(Manuscript received December 5, 2001; revision accepted for publication June 22, 2002.) 